University of Wollongong

Research Online

Australian Institute for Innovative Materials -

Papers

Australian Institute for Innovative Materials

$1-1-2016$

\title{
Mechanical stiffness augmentation of a 3D printed soft prosthetic finger
}

Rahim Mutlu

University of Wollongong, rmutlu@uow.edu.au

Solen Kumbay-Yildiz

University of Wollongong, solen@uow.edu.au

Gursel Alici

University of Wollongong, gursel@uow.edu.au

Marc in het Panhuis

University of Wollongong, panhuis@uow.edu.au

Geoffrey M. Spinks

University of Wollongong, gspinks@uow.edu.au

Follow this and additional works at: https://ro.uow.edu.au/aiimpapers

Part of the Engineering Commons, and the Physical Sciences and Mathematics Commons

Research Online is the open access institutional repository for the University of Wollongong. For further information contact the UOW Library: research-pubs@uow.edu.au 


\title{
Mechanical stiffness augmentation of a 3D printed soft prosthetic finger
}

\author{
Abstract \\ Soft robotics, as a multi-disciplinary research area, has recently gained a significant momentum due to \\ offering unconventional characteristics relative to rigid robots such as a resilient, highly dexterous, \\ compliant and safer interaction with humans and their physical environments. However, soft robots suffer \\ from not being able to carry their own weight which mainly depends on the modulus of elasticity of the \\ material used to fabricate them. In this paper, we report on a practical and easy-to-implement stiffness \\ augmentation method to enhance stiffness of soft robotic components. We fabricated a soft robotic \\ finger which is fully compliant with flexure hinges using Fused Deposition Modelling (FDM) technique and \\ a stiffness augmenting unit made of thin poly(vinyl chloride)(PVC) sheets. The stiffness of the entire \\ robotic finger was increased mechanically by linearly driving the stiffness augmenting unit. The \\ experimental data presented show that stiffness of the finger was increased by $40 \%$. Depending on the \\ material properties and thickness used for fabricating the stiffness augmenting unit, a higher rate of \\ stiffness increase can be easily obtained. \\ Disciplines \\ Engineering | Physical Sciences and Mathematics \\ Publication Details \\ Mutlu, R., Yildiz, S. Kumbay., Alici, G., in het Panhuis, M. \& Spinks, G. M. (2016). Mechanical stiffness \\ augmentation of a 3D printed soft prosthetic finger. Advanced Intelligent Mechatronics (AIM), 2016 IEEE \\ International Conference on (pp. 7-12). United States: IEEE.
}




\title{
Mechanical Stiffness Augmentation of a 3D Printed Soft Prosthetic Finger
}

\author{
Rahim Mutlu, S. Kumbay Yildiz, Gursel Alici", Marc in het Panhuis, and Geoff M. Spinks
}

\begin{abstract}
Soft robotics, as a multi-disciplinary research area, has recently gained a significant momentum due to offering unconventional characteristics relative to rigid robots such as a resilient, highly dexterous, compliant and safer interaction with humans and their physical environments. However, soft robots suffer from not being able to carry their own weight which mainly depends on the modulus of elasticity of the material used to fabricate them. In this paper, we report on a practical and easy-to-implement stiffness augmentation method to enhance stiffness of soft robotic components. We fabricated a soft robotic finger which is fully compliant with flexure hinges using Fused Deposition Modelling (FDM) technique and a stiffness augmenting unit made of thin poly(vinyl chloride)(PVC) sheets. The stiffness of the entire robotic finger was increased mechanically by linearly driving the stiffness augmenting unit. The experimental data presented show that stiffness of the finger was increased by $40 \%$. Depending on the material properties and thickness used for fabricating the stiffness augmenting unit, a higher rate of stiffness increase can be easily obtained.
\end{abstract}

\section{INTRODUCTION}

Soft robotics has gained formidable popularity among researchers particularly in the last few years. Soft robotics, as a sub-class of robotics, is a multi-disciplinary research area which not only interests roboticists but also researchers from other fields such as materials science, mechanical / electrical engineering, control engineering, chemistry, physics, computer science, biology, and medicine [1]. This emerging research area focuses on mechanisms, devices and robots partially or fully made of soft materials which provide those devices with compliance, high dexterity and more importantly adaptability to their working environments While a conventional robotic device made of rigid or highly stiff components is suitable for tasks requiring high precision and repeatability, soft robots are potential candidates for

R. Mutlu (rmutlu@uow.edu.au), G. Alici ('Corresponding Author gursel@uow.edu.au) and G.M. Spinks (gspinks@uow.edu.au) are with School of Mechanical, Materials and Mechatronic Engineering, and Intelligent Polymer Research Institute, ARC Centre of Excellence for Electromaterials Science, University of Wollongong, AIIM Facility, NSW, 2522, Australia.

S. K. Yildiz (solen@ee.hacettepe.edu.tr) is with the School of Mechanical, Material and Mechatronic Engineering, University of Wollongong, Wollongong, NSW 2522, Australia, on leave from the Department of Electrical and Electronics Engineering, Hacettepe University, 06800, Ankara, Turkey.

M. in het Panhuis (panhuis@uow.edu.au) is with the Soft Materials Group, School of Chemistry and Intelligent Polymer Research Institute, ARC Centre of Excellence for Electromaterials Science, University of Wollongong, AIIM Facility, NSW, 2522, Australia. applications such as unknown environments and where human presence exists due to their high compliance and shape conformation to the environment, and low stiffness.

Shape conforming robots, in other words, robotic manipulators with whole body manipulation capability were first introduced by Anderson and Horn [2]. It was called a robotic tentacle resembling an elephant trunk. Since then, there have been numerous studies on robotic manipulators under the name of hyper-redundant and/or continuum robots mimicking biological counterparts especially made of muscular systems such as elephant trunk [3, 4], octopus arm [5-7], snake [8-10], worm and caterpillar [11-13]. Following Hirose's innovative snake inspired robots [8], Chirikjian proposed modelling approaches to estimate kinematics and dynamics of hyper-redundant manipulators using backbone curve approach [14, 15]. While a hyper-redundant robotic manipulator resembles the biological counterpart using a high number of rigid links serially connected (a discrete robotic manipulator with a high degree of freedom), a continuum robotic manipulator forms natural-like shapes with its whole body in a continuous manner. Those studies are mainly a transition between conventional rigid robotics and soft robotics by partially consisting of rigid components covered with soft materials. Hyper-redundant and continuum robots have been proposed for areas such as minimally invasive surgery due to their capability to generate a large range of motions [16-18]. Hyper-redundant and continuum robots are usually actuated by a tendon system with a flexible backbone [19] or pneumatic actuators to generate shape deformation of the robotic manipulator [20].

Recent soft robotics studies have primarily focused on exploiting material properties, particularly flexible or soft materials, in order to build soft robots inspired from nature usually eliminating a backbone or an endoskeleton structure - a stiffer component [21]. Ilievski et al. have designed a soft actuation method using embedded pneumatic networks (PneuNets) in the soft structure of a starfish robot which generates complex motions when the channel network is pressurized [22]. Using the PneuNets, a number of bioinspired soft robots have been reported including fish [23], legged robot [24, 25] and hand rehabilitation [26]. Lin et al. studied a caterpillar soft robot actuated by shape memory alloys (working similar to tendon driven robotic systems) [13]. Even though soft robots have a number of advantages over rigid robots such as their compliance, dexterity and adaptability to surrounding objects, they suffer from not being able to carry their own weight due to the nature of the soft material used for their fabrication. Elastomeric polymer materials, mainly soft silicone rubbers, with low elastic moduli have been employed to fabricate 
these soft robots. On the other hand, biological systems are capable of changing their compliance (stiffness) due to the nature of their muscular hydrostat system.

Variable or tunable stiffness within a mechanism and/or machine has always been a desirable property. Early stiffness enhancing efforts for hyper-redundant and/or continuum manipulators are based on controlling the tension in the tendon-cables, if the manipulator is tendon-driven [27] or controlling the pressure in the air-muscles (e.g. McKibben), if the manipulator is pneumatically actuated; hence provide sufficiently low stiffness regulation [28]. Another actuation approach for pneumatically actuated soft robots is granular jamming which uses granules in the core of the soft robot and its stiffness is changed by de-pressurizing the core chamber $[29,30]$. Also a number of mechanical stiffness enhancement methods based on layer jamming have been studied. There are two major methods; one is based on de-pressurizing the chamber consisting of flexible layers [31] and the other type is based on mechanically tightening the layers [32]. Similar to granular jamming, the layer jamming method is also based on the friction between layers. When the contact area is increased by enfolding the flexible layers, stiffness of the entire structure is enhanced. It would be ideal to have an active material of the structure; hence variable stiffness would be integrated within the structure similar to natural muscles. Electroactive polymer (EAP) actuators are such engineered materials which show stiffness variation under an electrical input that can be directly used to enhance the stiffness of a soft robotic manipulator [33]. However, EAP actuators either generate a low stiffness or require a high electrical potential to actuate, depending on the type of the EAP material.

In this study, we present a practical method for mechanical stiffness augmentation of a bending type soft robotic manipulator, i.e. a $3 \mathrm{D}$ printed fully compliant robotic finger which is underactuated. Our approach uses thin and flexible PVC sheets within the soft robotic finger to augment its stiffness by linearly driving the PVC sheets through the channel designed in the soft robotic finger. This method is inspired by the layer jamming methods in order to control the stiffness of a soft robotic manipulator (our 3D printed soft robotic finger) to enhance the force output (grasping force) of the soft robotic finger. In this proposed method, there is no requirement for a pressure source to tighten the flexible layers or a servo drive to control the overlapping layer and friction. We manually change the position of the mechanical stiffness augmenting unit inside a designated channel within the soft finger. When thicker regions of the stiffness augmenting unit correspond to flexure hinges, stiffness of the soft finger increases.

\section{FABRICATION METHODS FOR SOFT RoBOTS}

As an emerging research area, there is no specific fabrication method to build a soft robot. However, a number of fabrication methods are used to construct the structure of a soft robot. These methods include mainly molding techniques [24, 34] and shape deposition manufacturing (SDM) [35] with a series of steps to fabricate the main body of the soft robot. As most of the soft robots reported in the literature have been fabricated from silicone rubbers, molding techniques or similarly SDM methods are quite suitable for the fabrication of a soft robot. The higher the complexity of the structure of the soft robot is, the harder and longer is the fabrication procedure for the soft robot, and certain design criteria are required in order to cast the desired shape. On the other hand, fabrication methods such as additive manufacturing are very promising not only for fabricating soft robots but also any object with a high geometrical complexity.

\section{A. Additive Manufacturing}

Additive manufacturing (AM) is a new term for rapid prototyping which dates back to 1960s. Elmqvist from Siemens [36] patented the first stream-line inkjet printing technology in 1951 which was originally called "3D Printing" using a powder material bound by an ink material [37]. However, 3D printing is nowadays used as a synonymous term for many additive manufacturing technologies including (but not limited to) fused deposition modelling (FDM), selective laser sintering (SLS), stereolithography (SLA) and powder bed fusion (PBF). Additive manufacturing is a paradigm shift from the subtractive manufacturing techniques such as casting/molding, machining, forging to the advanced manufacturing techniques that a final product is produced by adding a material layer-by-layer according to the digital design data of a real 3D object. Of additive manufacturing technologies, FDM has shown an exponential growth in the last decade due to its accessibility and comparatively low cost. The FDM method generally uses thermoplastic materials extruded layer-by-layer to form a 3D object.

\section{B. Materials}

The soft robotic finger studied in this paper has been fabricated from a commercially available thermoplastic elastomer (FilaFlex) which is a soft and stretchable material following curing. The main reasons for choosing this material are that it is ready to use and 3D printable with the FDM which has comparatively lower cost for prototyping a soft and compliant mechanism and/or body of a robotic device.

For the stiffness augmentation unit, we use a flexible sheet made of PVC with a thickness of $0.18 \mathrm{~mm}$ and has a higher stiffness compared to the thermoplastic (TPE) material used for printing the soft robotic finger.

\section{Design and Fabrication of the Soft Robotic Finger and Stiffness Augmentation Mechanism}

The soft robotic finger is designed as a monolithic body which works compliantly and silently, and is an underactuated system with three degrees of freedom (DoFs) corresponding to only one input. Rather than employing revolute joints which increase not only assembly requirements but also maintenance costs due to moving components, we use a fully-compliant mechanism approach in which the mechanism is fabricated from a single material with flexure hinges. A type of nonsymmetrical elliptic flexure hinge has been employed in the hinge design of the soft robotic finger [38]. The soft robotic finger is actuated with a tendon cable (inextensible braided string) connected to a servo motor. The tendon cable route is kept as close as possible to the free ends of flexure to apply a maximum 
bending moment to the flexure hinges. Of course, the ultimate desire is to use a soft material with built-in actuation (even further with integrated sensing) which will eliminate all the external components and additional assembly for the actuation unit, and the structural material would provide a monolithic body with actuating as well as sensing all in one unit [39, 40]. Furthermore, a prismatic channel has been included in the design of the soft robotic finger to house the stiffness augmenting unit. The stiffness augmenting unit is designed, corresponding to the geometrical parameters of the soft robotic finger especially with reference to location of the flexure hinges.

While the stiffness enhancement can be performed by moving the stiffness augmenting unit forward or backward depending on the preference, we have used a forward design that the PVC sheet is pushed towards the tip of the finger to increase the entire finger's stiffness. Figure 1 shows the cross sectional view of the soft robotic finger with channels and the stiffness augmenting unit with corresponding dimensions.

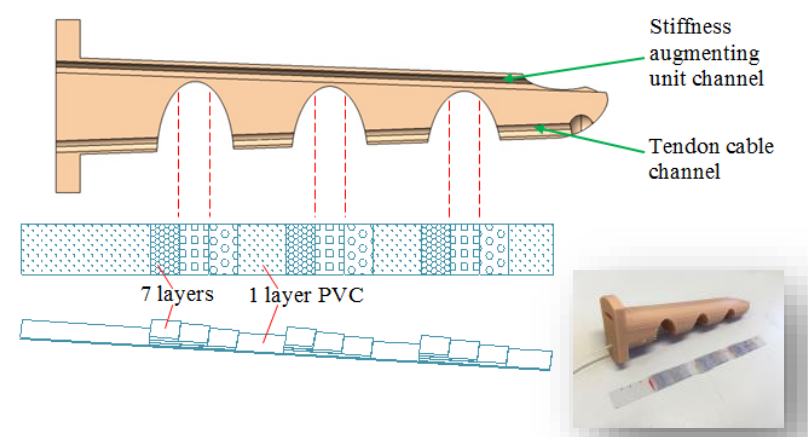

Figure 1. Cross sectional view along the soft robotic finger and the stiffness augmenting unit (photo of the real finger with stiffness augmenting unit is also presented in the bottom right corner).

We have used the FDM based additive manufacturing method to fabricate the soft robotic finger as a monolithic structure working compliantly due to its flexure hinges. As a 3D printer, UP Plus 2 from 3D Printing Systems has been employed. We modified and optimized the extruding unit and printing parameters of the 3D printer in order to extrude the TPE material. The AM techniques are in general used either for prototyping or the purpose of customization which would be cost effective, compared to traditional manufacturing methods. The soft robotic finger is designed with a prismatic channel passing along the finger through flexure hinges in which the stiffness augmenting unit can be inserted and freely moves. The $3 \mathrm{D}$ printing platform and the soft robotic finger with the stiffness augmenting unit are presented in Figure 2. A braided inextensible string has been used to complete the soft robotic finger to be actuated with a servo motor.

The stiffness augmenting unit was made of a thin PVC sheet which is precisely cut into the dimension corresponding to the prismatic channel within the soft robotic finger. A laser engraving machine (Universal Laser Systems, Model: VLS 3.50) was used to cut the PVC sheets into predetermined sizes. The stiffness augmentation unit was prepared by placing the layers of the laser cut PVC sheets and bonding them at one end to give free movement on the other end when bending occurs within the flexure hinges during the operation of the soft robotic finger. As a proof of concept, four incremental stiffness enhancing regions have been designed where the numbers of PVC layers are 1, 3, 5 and 7. It must be noted that this method is not restricted to any number of layers but depends on the stiffness augmentation requirements and elastic properties of the material. By varying the number of incremental steps used in the stiffness augmenting unit, the rate of stiffness augmentation can be modified.

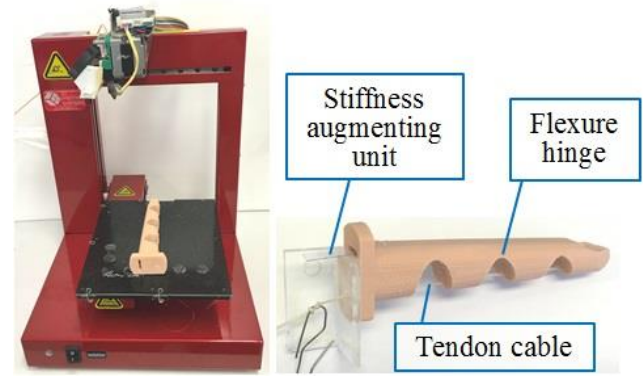

Figure 2. A typical fully compliant soft robotic finger on the 3D printing platform (on the left) and the finger assembly with a tendon cable and the stiffness augmentation mechanism (on the right).

\section{EXPERIMENTAL RESULTS AND DISCUSSION}

Experiments were conducted in order to determine the stiffness enhancement capability of the proposed stiffness augmenting mechanism within the soft robotic finger. As mentioned, a tendon driven actuation method has been used to actuate (flex) the soft robotic finger and the tension force in the tendon cable was measured for each test. Stiffness augmenting unit has four incremental steps, i.e. 1, 3, 5 and 7 layers of $0.18 \mathrm{~mm}$ PVC sheets. Starting with 1-layer PVC sheet as a default position for the stiffness augmenting unit in the soft robotic finger, there are three steps of stiffness augmentation in this design. The stiffness augmenting unit was incrementally driven (slid) forward towards the tip of the soft robotic finger in the prismatic channel and the corresponding tension force (input force) required to flex the soft robotic finger was measured.

\section{A. Experimental setup}

The experimental setup consists of a number of components including two stage platforms; one stage is a platform to place and fix the finger and the second stage is a micrometer positioning stage with a servo motor connected to the micrometer to drive the stage. We used a force sensor (Lutron FG-5005) which was fixed on the micrometer positioning stage and the tendon cable of the soft robotic finger was connected to the force sensor. The stiffness augmenting unit was incrementally driven in the soft robotic finger to predetermined positions, which corresponded to three flexure hinge locations in the soft robotic finger. Flexion of the finger from relaxed (straight) to fully bent state was recorded with a digital camera (Nikon D5100) at $30 \mathrm{fps}$. The experimental setup is shown in Figure 3. We used an image processing algorithm developed in MATLAB in order to determine position, force and time relation of the soft robotic finger during its flexion motion. Image frames were extracted from the recorded videos of each experiment and were analyzed to obtain the data. 


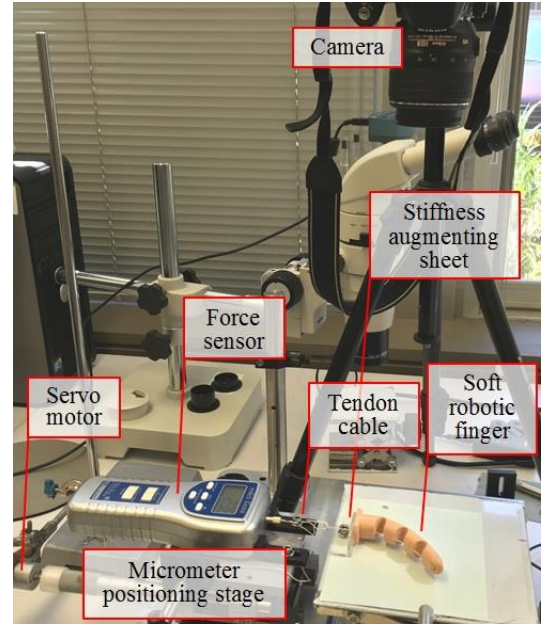

Figure 3. Experimental setup.
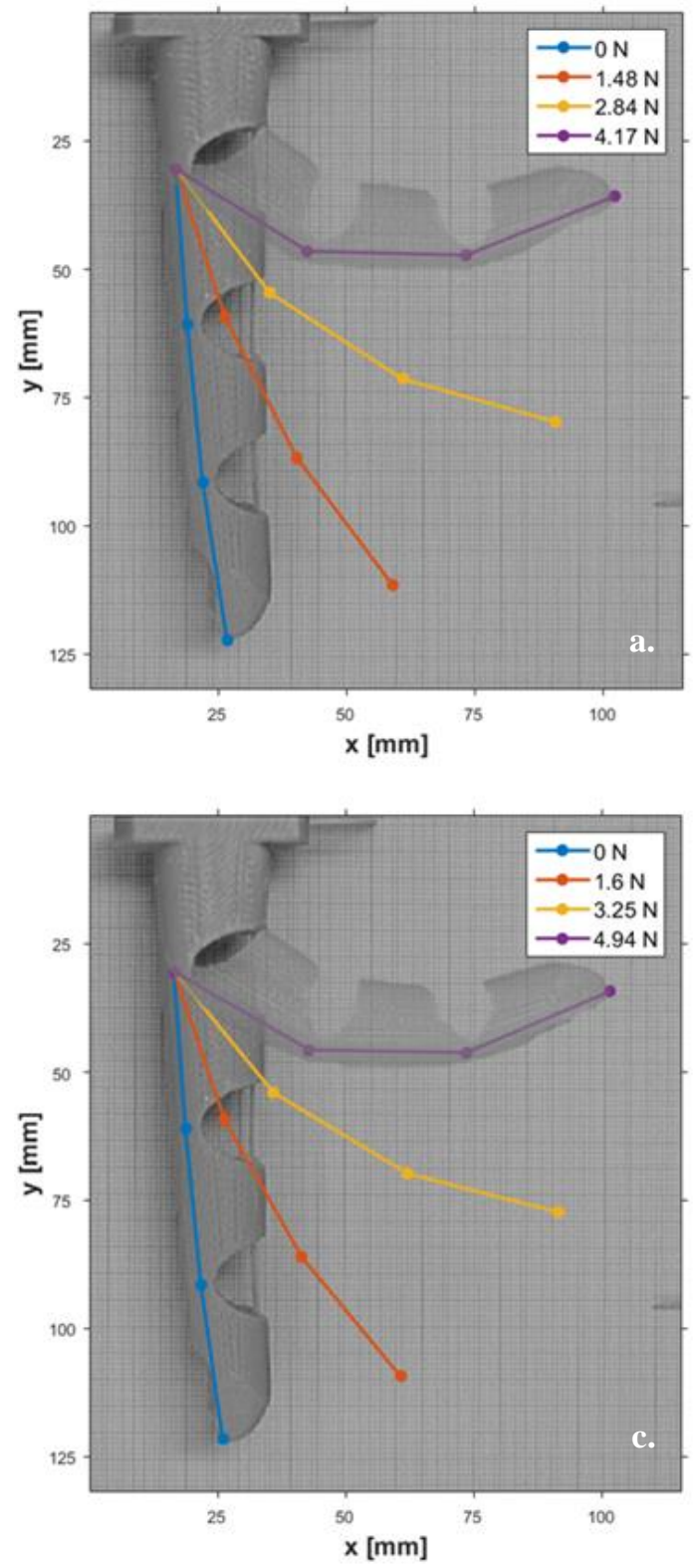

Flexure hinge positions, as joint coordinates, were obtained from the analyzed image frames and the corresponding tension force at the tendon cable of the finger. For the sake of brevity, three steps are plotted to show the tension force required to bend the soft robotic finger with a different stiffness from a relaxed state to a fully bent state. The tension force results and corresponding soft finger flexion configurations under different stiffness augmentations (1 layer, 3, 5 and 7 layers of PVC sheet) are provided in Figure 4. Corresponding tension force results are presented in Figure 5. For the purpose of evaluating stiffness enhancing results of the proposed mechanism, the stiffness variations are presented using the displacement versus force relation for the actuating tendon cable.
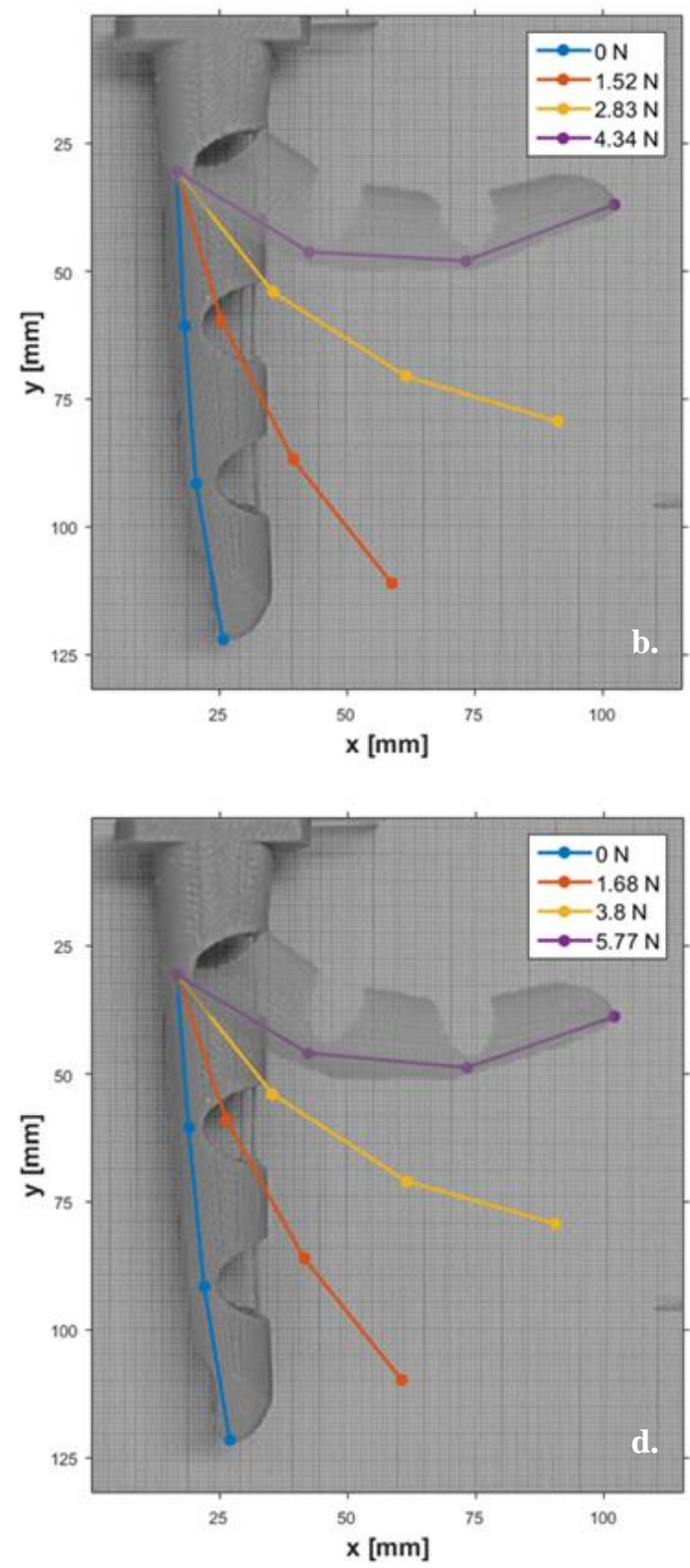

Figure 4. The soft robotic finger flexion steps with stiffness augmentation (finger is at its initial and bent (transparent) states at the background) using a. 1 layer, b. 3 layers, c. 5 layers and d. 7 layers of PVC sheet. 


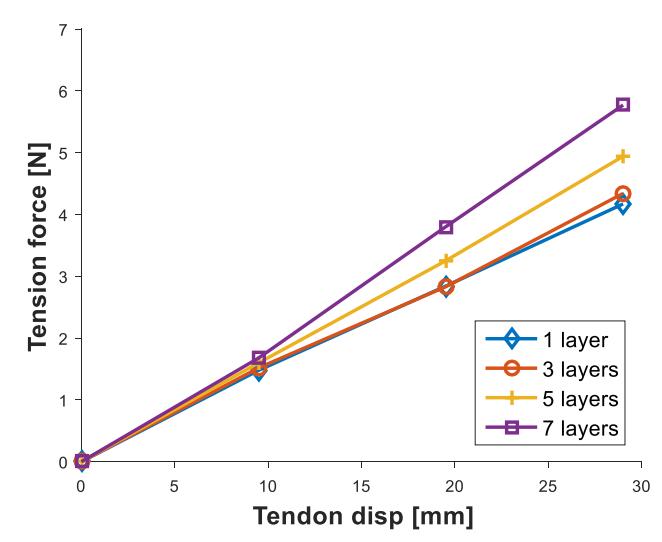

Figure 5. Tensile force results for the stiffness augmenting unit with various thickness; from a relaxed to a fully flexed state.

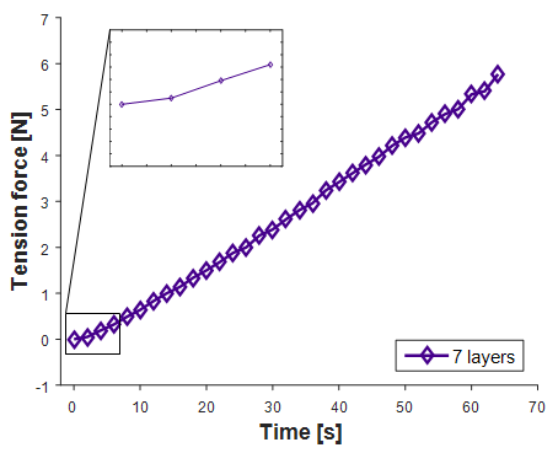

Figure 6. Tensile force results for a 7-layer stiffness augmenting unit; from a relaxed to a fully flexed state with zoomed view (time is linearly proportional to the tendon displacement).

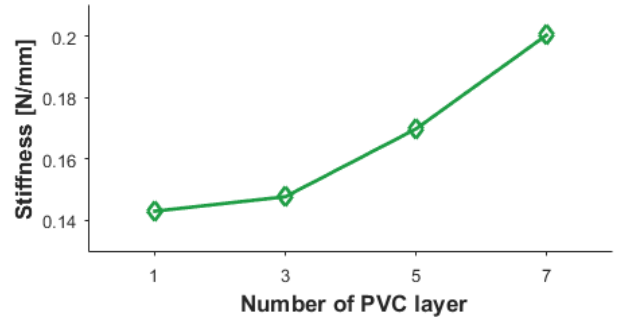

Figure 7. Stiffness of a soft robotic finger with varying number of PVC layers in the stiffness augmenting unit.

As evident from Figure 5, a stiffness increase up to $40 \%$ is accomplished by increasing the number of layers in the stiffness augmentation unit from 1 to 7 at the fully bent state of the soft robotic finger. Stiffness of the soft finger is quite linear as shown in Figure 6. Initially, the tensile force shows a non-linear behavior which may be due to the gap between the stiffness augmenting sheet and the channel in the soft robotic finger. The rest of the flexion motion of the soft robotic finger with stiffness augmentation is linearly proportional to time or tendon displacement as the tendon cable is driven linearly. Using linear fits for the tensile force over the tendon cable displacements, the stiffness of the whole soft robotic finger for various stiffness augmenting layers is calculated and presented in Figure 7. While number of PVC layer increases linearly, stiffness increase shows a nonlinear behavior. This might be due to the amount of bonding material used for stacking PVC layers. Also, it must be noted that, this stiffness augmentation design is not restricted to one material or thickness of the sheet, but any flexible or soft material can be used for stiffness enhancement criteria. In addition, various materials can be used within the stiffness augmentation unit to design the stiffness variation profile. For instance, a flexible PVC material can be used only as the base layer and a thin stainless steel layer can be used for other layers with different thicknesses which would provide initially a softer stiffness increase and then a sharper increase when more layers are in effect.

\section{CONCLUSION AND FUTURE WORK}

A fully compliant soft robotic finger with three flexure hinges has been designed and fabricated using a FDM based $3 \mathrm{D}$ printing method and a thermoplastic elastomer material. This type of AM technique has been employed for the purpose of prototyping as well as suitability for customization which is comparatively cost effective in consideration of $3 \mathrm{D}$ printing unit cost. A stiffness augmenting mechanism was made using thin $(0.18 \mathrm{~mm})$ and flexible PVC sheets with varying number of layers. A single layer of the PVC sheet was used as the base layer and three incremental stiffness varying regions were made by bonding the PVC sheets (cut accordingly to the flexure hinge locations) at their one end and the other ends are left free to move while the soft robotic finger is bending. The stiffness augmenting mechanism is based on the mechanical stiffness enhancement where thicker layers provide higher stiffness to the entire soft robotic finger. Resulting stiffness levels of the soft robotic finger were quantitatively compared. The soft robotic finger was actuated through a tendon cable attached to a force sensor which was also fixed to the micrometer stage driven by a servo motor.

The stiffness augmenting mechanism increases the stiffness of the entire soft robotic finger: a $40 \%$ increase is obtained with 7-layers of PVC sheets in comparison to the base PVC layer (1-layer). As a proof of concept, the proposed stiffness augmentation method is easy to implement and suitable to be used with a number of augmentation units with different stiffness variation profiles.

Future work will include a comparison of other materials which are flexible and suitable to use within the soft robotic finger or any soft robotic device with the aim of stiffness increase in the entire soft robot. We also plan to use a combination of various materials to determine and compare their effect on the stiffness of the soft robotic finger. Furthermore, a comparatively much stiffer material can be used to lock the soft robotic finger from soft to rigid-like state and employ the finger in a soft prosthetic hand device to improve its grasping force.

\section{ACKNOWLEDGMENTS}

This work has been supported by ARC Centre of Excellence for Electromaterials Science (Grant No. CE140100012) and the University of Wollongong. S. Kumbay Yildiz acknowledges the support provided by The Scientific and Technological Research Council of Turkey (TÜBİTAK) 
under the 2219 - Postdoctoral Research Scholarship Program.

\section{REFERENCES}

[1] L. Margheri and B. Trimmer, "Soft Robotics Community Events: Meeting Different Backgrounds for Common Challenges," Soft Robotics, vol. 1, pp. 236-238, 2014.

[2] (1968). Minsky-Bennett Tentacle. Available: http://cyberneticzoo.com/underwater-robotics/1968-minsky-bennettarm-marvin-minsky-and-bill-bennett-american/

[3] M. W. Hannan and I. D. Walker, "Kinematics and the Implementation of an Elephant's Trunk Manipulator and Other Continuum Style Robots," Journal of Robotic Systems, vol. 20, pp. 45-63, 2003.

[4] O. Salomon and A. Wolf, "Inclined Links Hyper-Redundant Elephant Trunk-Like Robot," Journal of Mechanisms and RoboticsTransactions of the Asme, vol. 4, pp. 045001-045001-6, Nov 2012.

[5] R. Kang, A. Kazakidi, E. Guglielmino, D. T. Branson, D. P. Tsakiris, J. A. Ekaterinaris, et al., "Dynamic model of a hyper-redundant, octopus-like manipulator for underwater applications," in Intelligent Robots and Systems (IROS), 2011 IEEE/RSJ International Conference on, 2011, pp. 4054-4059.

[6] C. Laschi, B. Mazzolai, V. Mattoli, M. Cianchetti, and P. Dario, "Design of a biomimetic robotic octopus arm," Bioinspiration \& Biomimetics, vol. 4, p. 015006, 2009.

[7] Y. Yekutieli, R. Sagiv-Zohar, R. Aharonov, Y. Engel, B. Hochner, and T. Flash, "Dynamic Model of the Octopus Arm. I. Biomechanics of the Octopus Reaching Movement," Journal of Neurophysiology, vol. 94, pp. 1443-1458, 2005.

[8] S. Hirose and M. Mori, "Biologically Inspired Snake-like Robots," in Robotics and Biomimetics, 2004. ROBIO 2004. IEEE International Conference on, 2004, pp. 1-7.

[9] B. Atakan, A. M. Erkmen, and I. Erkmen, "3-D grasping during serpentine motion with a snake-like robot," in Proceedings of the Sixth IASTED Int Conf. on Robotics and Applications, 2005, pp. 46-51.

[10] C. D. Onal and D. Rus, "A modular approach to soft robots," in Biomedical Robotics and Biomechatronics (BioRob), 2012 4th IEEE RAS \& EMBS International Conference on, 2012, pp. 1038-1045.

[11] S. Sangok, C. D. Onal, C. Kyu-Jin, R. J. Wood, D. Rus, and K. Sangbae, "Meshworm: A Peristaltic Soft Robot With Antagonistic Nickel Titanium Coil Actuators," Mechatronics, IEEE/ASME Transactions on, vol. 18, pp. 1485-1497, 2013.

[12] S. M. Felton, M. T. Tolley, C. D. Onal, D. Rus, and R. J. Wood, "Robot Self-Assembly by Folding: A Printed Inchworm Robot," in 2013 IEEE International Conference on Robotics and Automation (ICRA), 2013, pp. 277-282.

[13] L. Huai-Ti, G. L. Gary, and T. Barry, "GoQBot: a caterpillar-inspired soft-bodied rolling robot," Bioinspiration \& Biomimetics, vol. 6, p. 026007,2011

[14] G. S. Chirikjian and J. W. Burdick, "Kinematically optimal hyperredundant manipulator configurations," Robotics and Automation, IEEE Transactions on, vol. 11, pp. 794-806, 1995.

[15] G. S. Chirikjian, "A continuum approach to hyper-redundant manipulator dynamics," in Intelligent Robots and Systems, IROS '93. Proceedings of the 1993 IEEE/RSJ International Conference on, 1993, pp. $1059-1066$

[16] D. B. Camarillo, T. M. Krummel, and J. K. Salisbury Jr, "Robotic technology in surgery: Past, present, and future," The American Journal of Surgery, vol. 188, pp. 2-15, 2004.

[17] T. Ota, A. Degani, D. Schwartzman, B. Zubiate, J. McGarvey, H. Choset, et al., "A novel highly articulated robotic surgical system for epicardial ablation," in Engineering in Medicine and Biology Society, 2008. EMBS 2008. 30th Annual International Conference of the IEEE, 2008, pp. 250-253.

[18] T. Kato, I. Okumura, S.-E. Song, and N. Hata, "Multi-section continuum robot for endoscopic surgical clipping of intracranial aneurysms," MICCAI Int. Conf. on Medical Image Computing and Computer-Assisted Intervention, vol. 16, pp. 364-371, 2013.

[19] M. W. Hannan and I. D. Walker, "Analysis and experiments with an elephant's trunk robot," Advanced Robotics, vol. 15, pp. 847-858, 2001.
[20] D. Trivedi, C. D. Rahn, W. M. Kier, and I. D. Walker, "Soft robotics: Biological inspiration, state of the art, and future research," Applied Bionics and Biomechanics, vol. 5, pp. 99-117, 2008.

[21] R. F. Shepherd, F. Ilievski, W. Choi, S. A. Morin, A. A. Stokes, A. D. Mazzeo, et al., "Multigait soft robot," Proceedings of the National Academy of Sciences, vol. 108, pp. 20400-20403, 2011.

[22] F. Ilievski, A. D. Mazzeo, R. F. Shepherd, X. Chen, and G. M. Whitesides, "Soft Robotics for Chemists," Angewandte Chemie International Edition, vol. 50, pp. 1890-1895, 2011.

[23] A. D. Marchese, C. D. Onal, and D. Rus, "Autonomous Soft Robotic Fish Capable of Escape Maneuvers Using Fluidic Elastomer Actuators," Soft Robotics, vol. 1, pp. 75-87, 2014.

[24] M. T. Tolley, R. F. Shepherd, B. Mosadegh, K. C. Galloway, M. Wehner, M. Karpelson, et al., "A Resilient, Untethered Soft Robot," Soft Robotics, vol. 1, pp. 213-223, 2014.

[25] J. M. Florez, B. Shih, Y. Bai, and J. K. Paik, "Soft pneumatic actuators for legged locomotion," in Robotics and Biomimetics (ROBIO), 2014 IEEE International Conference on, 2014, pp. 27-34.

[26] P. Polygerinos, S. Lyne, W. Zheng, L. F. Nicolini, B. Mosadegh, G. M. Whitesides, et al., "Towards a soft pneumatic glove for hand rehabilitation," in Intelligent Robots and Systems (IROS), 2013 IEEE/RSJ International Conference on, 2013, pp. 1512-1517.

[27] Y.-J. Kim, S. Cheng, S. Kim, and K. Iagnemma, "A stiffnessadjustable hyperredundant manipulator using a variable neutral-line mechanism for minimally invasive surgery," Robotics, IEEE Transactions on, vol. 30, pp. 382-395, 2014

[28] W. McMahan, V. Chitrakaran, M. Csencsits, D. Dawson, I. D. Walker, B. A. Jones, et al., "Field trials and testing of the OctArm continuum manipulator," in Robotics and Automation, ICRA 2006. Proceedings 2006 IEEE International Conference on, 2006, pp. 2336-2341.

[29] A. Jiang, G. Xynogalas, P. Dasgupta, K. Althoefer, and T. Nanayakkara, "Design of a variable stiffness flexible manipulator with composite granular jamming and membrane coupling," in Intelligent Robots and Systems (IROS), 2012 IEEE/RSJ International Conference on, 2012, pp. 2922-2927.

[30] M. Cianchetti, T. Ranzani, G. Gerboni, I. D. Falco, C. Laschi, and A. Menciassi, "STIFF-FLOP surgical manipulator: Mechanical design and experimental characterization of the single module," in Intelligent Robots and Systems (IROS), 2013 IEEE/RSJ International Conference on, 2013, pp. 3576-3581

[31] Y. J. Kim, S. Cheng, S. Kim, and K. Iagnemma, "Design of a tubular snake-like manipulator with stiffening capability by layer jamming," in Intelligent Robots and Systems (IROS), 2012 IEEE/RSJ International Conference on, 2012, pp. 4251-4256.

[32] J. L. C. Santiago, I. D. Walker, and I. S. Godage, "Continuum robots for space applications based on layer-jamming scales with stiffening capability," in Aerospace Conference, 2015 IEEE, 2015, pp. 1-13.

[33] R. Mutlu and G. Alici, "Artificial muscles with adjustable stiffness," Smart Materials and Structures, vol. 19, p. 045004 (10pp), 2010.

[34] S. Yi, S. Yun Seong, and J. Paik, "Characterization of silicone rubber based soft pneumatic actuators," in Intelligent Robots and Systems (IROS), 2013 IEEE/RSJ International Conf on, 2013, pp. 4446-4453.

[35] J. Gafford, Y. Ding, A. Harris, T. McKenna, P. Polygerinos, D. Holland, et al., "Shape Deposition Manufacturing of a Soft, Atraumatic, Deployable Surgical Grasper1," Journal of Medical Devices, vol. 8, pp. 030927-030927, 2014.

[36] R. Elmqvist, "Measuring instrument of the recording type," U.S. Patent 2566443, 1951.

[37] E. Sachs, M. Cima, and J. Cornie, "Three-Dimensional Printing: Rapid Tooling and Prototypes Directly from a CAD Model," CIRP Annals Manufacturing Technology, vol. 39, pp. 201-204, 1990.

[38] R. Mutlu, G. Alici, M. in het Panhuis, and G. Spinks, "Effect of flexure hinge type on a 3D printed fully compliant prosthetic finger," in Advanced Intelligent Mechatronics (AIM), 2015 IEEE International Conference on, 2015, pp. 790-795.

[39] R. Mutlu, G. Alici, X. Xiang, and W. Li, "An active-compliant microstage based on EAP artificial muscles," in Advanced Intelligent Mechatronics (AIM), 2014 IEEE/ASME International Conference on, 2014, pp. 611-616

[40] R. Mutlu, G. Alici, and W. Li, "A Soft Mechatronic Micro-Stage Mechanism Based on Electroactive Polymer Actuators," IEEE/ASME Transactions on Mechatronics, Vol.21, No.3, pp.1467 -- 1478, June 2016 
